Article

\title{
Preparation and surface characterization of nanodisk/nanoflower-structured gallium-doped zinc oxide as a catalyst for sensor applications
}

\author{
R. Jothi Ramalingam a,c,*, T. Radika b, Hamad A. Al-Lohedan a \\ a Surfactant Research Chair, Chemistry Department, College of Science, King Saud University, Riyadh 11451, Saudi Arabia \\ b Centre for Materials for Electronics Technology, Govt. of India, Thrissur, Kerala, India \\ c School of Engineering, University of Ulsan, Korea
}

\section{A R T I C L E I N F O}

Article history:

Received 24 January 2016

Accepted 9 May 2016

Published 5 August 2016

Keywords:

Semiconductors

Thin films

Sol-gel growth

Atomic force microscopy

Electrical conductivity

Surface property

\begin{abstract}
A B S T R A C T
Nanostructured gallium-doped zinc oxide (GZO) thin films were fabricated on piezoelectric substrates. The GZO thin films with nanodisk/nanoflower morphologies were prepared by a simple spin-coating process followed by one-step hydrothermal treatment. Addition of polymer during hydrothermal treatment resulted in nanodisk and nanoflower morphologies. The morphology, microstructure and chemical composition of thin films prepared under different conditions were examined by field-emission scanning electron microscopy (FE-SEM), X-ray diffraction (XRD) and Raman spectroscopy. The XRD and FE-SEM investigations confirmed that the GZO nanodisks, nanorods and nanoflowers formed on the AlN/Si substrates were all wurtzite phase. Green fluorescent protein (GFP) was immobilized on the as-synthesized GZO nanostructured materials by a dipping process. Atomic force microscopy (AFM) and fluorescence spectroscopy measurements were conducted to confirm the surface binding nature of GFP on the GZO nanostructures to determine their suitability for use in sensor applications and bioimaging techniques. Trace-level addition of GFP to the GZO nanostructures resulted in a fluorescence response, revealing good activity for ultraviolet light sensor applications.
\end{abstract}

(C) 2016, Dalian Institute of Chemical Physics, Chinese Academy of Sciences. Published by Elsevier B.V. All rights reserved.

\section{Introduction}

Gallium-doped zinc oxide (GZO)-based nanostructured thin films have recently been prepared by different methods and applied in important research areas such as photocatalysis, sensors and dye-sensitized solar cells [1-4]. Nanocomposites of $\mathrm{ZnO}$ are providing better results than pristine $\mathrm{ZnO}$ or $\mathrm{ZnO/graphene.} \mathrm{For} \mathrm{example,} \mathrm{graphene} \mathrm{decorated} \mathrm{with} \mathrm{manga-}$ nese-doped ZnO nanoparticles exhibited enhanced visible-light photocatalytic activity for industrial textile waste water treatment [5]. However, it is challenging to form ordered nanostructures to achieve higher activity in catalysis and solar cell applications [2,3]. The biological properties of protein-immobilized $\mathrm{ZnO}$ and GZO nanostructures have not been studied in detail. ZnO nanomaterials in the form of quantum dots (QDs) or nanoparticles are potentially applicable in biological cell-labeling and sensor applications [6-8]. GZO nanorods and nanostructured materials have also shown promise for use in the fields of light-emitting diodes and optical devices [8]. The main advantage of GZO and undoped $\mathrm{ZnO}$ is that they are relatively nontoxic compared with other semiconductor materials like cadmium-based compounds, which are harmful

\footnotetext{
* Corresponding author. Tel: +966-553510565; Fax: +966-1-4679972; E-mail: jrajabathar@ksu.edu.sa 
to human beings and the environment because they do not biodegrade [7]. Nanostructured ZnO materials are also attractive for application in gas sensors and biosensing because of their high aspect ratio, polar surface along the $c$-axis, and high electron mobility. Notably, the isoelectric point of $\mathrm{ZnO}$ is high (around 9.5), which is favorable for immobilization of biological species with low isoelectric point, such as enzymes and proteins, assisted by electrostatic attraction at suitable $\mathrm{pH}$ [9].

In the past decades, $\mathrm{ZnO}$ nanomaterials with different morphologies like porous films, nanoparticles, and nanorods have been developed for biosensing applications involving detection of proteins, uric acid, cytochrome c, [10-15], glucose [16,17], and phenolic compounds [18]. An advantage of doping the $\mathrm{ZnO}$ lattice with $\mathrm{Ga}$ is that it causes the electron conductivity of $\mathrm{ZnO}$ to increase by three times compared with that of pristine $\mathrm{ZnO}$ [19]. In 2013, Jothi Ramalingam's group [19] fabricated GZO nanodisks by a spin coating process followed by hydrothermal treatment with polymer assistance. Nanodisk formation depended on the preparation methodology and presence of an aluminum coating on the piezoelectric substrate. Inorganic nanomaterials with added polymer are important nanocomposites to develop low-cost sensor devices for environmental gas monitoring. Some of these nanomaterials possess specific properties such as piezoelectricity, which has allowed the development of transducers using either surface or bulk acoustic waves to measure perturbation in fundamental frequencies by the added mass on their surface. Recent biosensor research indicates that the crystalline form of $\mathrm{SiO}_{2}$, a piezoelectric crystal with an inert surface, is a potential candidate for the immobilization of proteins for biodetection [20]. Recently, Xu et al. [21] reported a photoelectrochemical electrode containing $\mathrm{ZnO}$ with an inverse opal structure for $\alpha$-fetoprotein (AFP) detection and glucose oxidase (GOD) sensor applications. Uniform CdS QDs were synthesized by a hydrothermal method followed by binding of AFP and GOD to form an AFP-CdS-GOD composite. A competitive immunosensor consisting of AFP and the AFP-CdS-GOD composite with anti-AFP antibodies immobilized on an FTO/ZnO electrode was used to detect AFP molecules. The CdS QDs broadened the absorption range of visible light and GOD acted as a catalyst for glucose, providing electrons and increasing the photocurrent. The developed immunoassay achieved high sensitivity for AFP. In another example, Brince Paul et al. [22] used a self-assembled monolayer-modified copper-doped $\mathrm{ZnO}$ nanofiber interface to detect plasmodium falciparum histidine-rich protein-2 with the goal of diagnosis of malarial infections. Incorporation of copper into $\mathrm{ZnO}$ not only increased the conductivity of the nanofibers but also pre-concentrated the target analyte (protein) onto the nanofiber surface because of the inherent electric field produced at the $\mathrm{CuO} / \mathrm{ZnO}$ heterojunction interface.

The present study describes preparation methodology to make GZO nanostructures like nanodisks and nanoflowers with good conductivity. The nanostructures are characterized by various surface techniques like X-ray diffraction (XRD), field-emission scanning electron microscopy (FE-SEM), atomic force microscopy (AFM) and Raman spectroscopy. Green fluorescent protein (GFP) is immobilized on the GZO nanodisk and nanoflower materials, as confirmed by florescence spectroscopy. The prepared materials show promising activity as sensors in the presence of UV irradiation.

\section{Experimental}

\subsection{Preparation of GZO thin films}

GZO coating solutions were prepared by mixing zinc acetate dihydrate, methoxyethanol, gallium(III) nitrate, and monoethanolamine, which were A. R. grade and used without further purification. Appropriate quantities of the precursors were stirred with a magnetic stirrer at $70{ }^{\circ} \mathrm{C}$ for $2 \mathrm{~h}$. Polyethylenimine (non-ionic) polymer solution was purchased from TCI chemical ( $30 \%$ dissolved in water). GZO containing (1, 2 and 3 ) mol\% gallium, which is designated as $1 \%$ GZO, $2 \%$ GZO and $3 \%$ GZO, respectively, was prepared by a reported procedure [19]. The prepared GZO solutions were coated on AlN/Si substrates by spin coating at $1000-2500 \mathrm{r} / \mathrm{min}$. After each deposition, the films were heated on a heating plate at $300^{\circ} \mathrm{C}$ for $10 \mathrm{~min}$. Four to six layers of each GZO thin film were deposited to obtain a thickness of 300-350 nm. Following spin coating, the samples were annealed in a furnace at $500{ }^{\circ} \mathrm{C}$ for $3 \mathrm{~h}$.

\subsection{GZO nanostructure formation by the hydrothermal method}

The GZO films on AlN/Si substrates were hydrothermally treated in the presence of $3 \%$ polymer solution to fabricate GZO nanodisks or nanoflowers. GZO nanorods as a reference compound were prepared by performing the hydrothermal process without adding polymer solution. In the hydrothermal process, equal volumes of zinc nitrate and hexamethylenetetramine solutions $(40 \mathrm{~mL})$ were mixed with $3 \%$ polymer solution $(10 \mathrm{~mL})$ for a few minutes, and then the mixture was transferred into a Teflon-lined autoclave. A 1\% GZO film on an AlN/Si substrate was fixed vertically in a holder inside the autoclave. Nanodisk structures were grown at $90{ }^{\circ} \mathrm{C}$ for $20 \mathrm{~h}$. In the case of nanoflower formation, the GZO-coated substrate was kept in a horizontal position instead of vertical inside the Teflon-lined autoclave. The same procedure was repeated using 2\% GZO- and 3\% GZO-coated substrates.

\subsection{Protein immobilization on GZO nanostructures}

A solution of GFP (5 $\mu \mathrm{mol} / \mathrm{L}, 1 \mathrm{~mL})$ in Tris-HCl buffer medium ( $\mathrm{pH}=7.0$ ) was incubated at $37^{\circ} \mathrm{C}$ with a GZO-coated substrate in a 5-mL vessel for time intervals ranging from 15 to 60 min. The substrates were dried at room temperature for further analysis. The structure and morphology of the substrates were characterized by XRD and FE-SEM. The crystalline phases of the samples were analyzed by XRD analysis with a Rigaku ultra-X diffractometer ( $\mathrm{Cu} K_{\alpha}$ radiation, $40 \mathrm{kV}, 120 \mathrm{~mA}$ ). FE-SEM and energy-dispersive X-ray spectroscopy (JSM-6500F, JEOL) and AFM (PARK SYSTEM XE 100 E) were used for surface analysis. Raman spectroscopic characterization (Confocal Raman Microscope alpha 300R) was conducted with a laser energy source of $532 \mathrm{~nm}$ at a fixed temperature. An Olympus 
microscope was connected to the Raman instrument to record the corresponding microscopic images. WITec software was used to assign peak values. Fluorescence spectra of control and GFP-immobilized GZO samples were recorded on a spectrofluorimeter (RF-5301PC, Shimadzu, Japan). Electrical conductivity and Keithley 4200-SCS semiconductor characterization equipment were used to study the sensing activities of the fabricated thin-film materials.

\section{Results and discussion}

XRD patterns of GZO samples with different morphology are shown in Fig. 1 . The $2 \theta$ values of the respective major peaks (assignments in brackets) were observed at $2 \theta=31.8^{\circ}(100)$, $34.5^{\circ}$ (002), $36.3^{\circ}$ (101), $47.6^{\circ}(102)$, and $56.6^{\circ}$ (110) for GZO nanoflower sample and $2 \theta=31.7^{\circ}(100), 34.3^{\circ}(002), 36.1^{\circ}$ (101), $47.4^{\circ}(102)$, and $56.5^{\circ}$ (110) for GZO nanodisk sample. GZO displayed a characteristic intense peak at $2 \theta=43.4^{\circ}(002)$. The crystallite sizes of GZO nanoflowers and nanodisks were determined from the full width at half-maximum of each peak using the Scherrer formula. The intensity of the major peaks in the XRD patterns are clearly different for the nanodisk and nanoflower morphologies. The (100) plane is the most intense peak for the nanoflower morphology, with a crystallize size of $\sim 77.8 \mathrm{~nm}$. In contrast, the (101) plane is the most intense peak for the nanodisk morphology, with a corresponding crystallite size of $\sim 56.1 \mathrm{~nm}$. The XRD results clearly reflect the differences in their crystalline properties and nanostructures of the GZO samples.

We studied the role of the concentration of the GZO solution, which is the initial precursor for $\mathrm{ZnO}$ seed layer formation, in the formation of fine nanodisk morphology in our previous report [19]. The optimized concentration of GZO (0.5 mol/L)

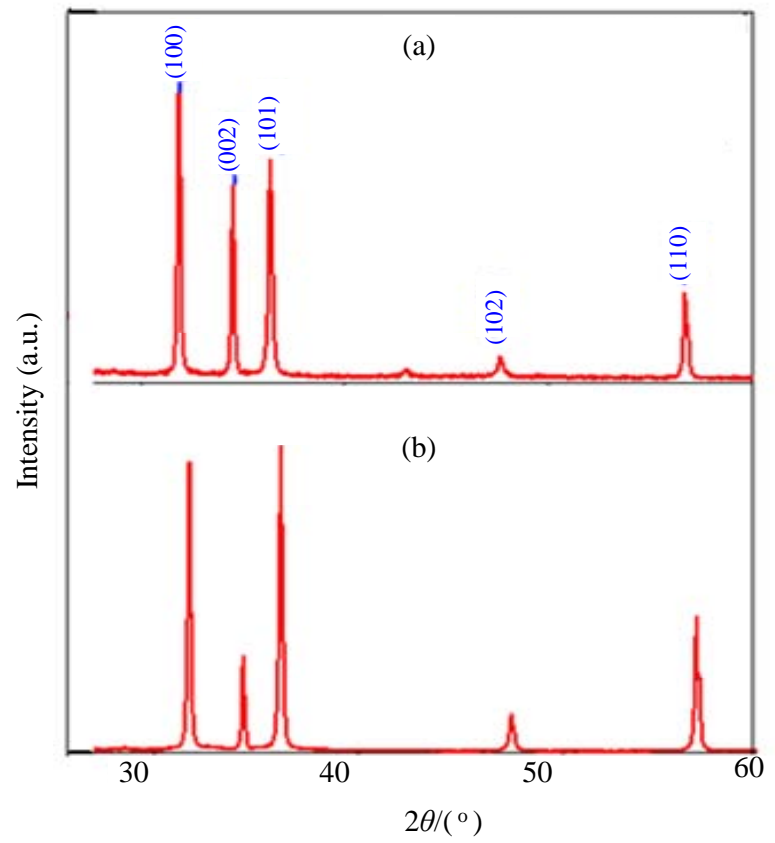

Fig. 1. XRD patterns of GZO nanoflower (a) and GZO nanodisk (b) structures.

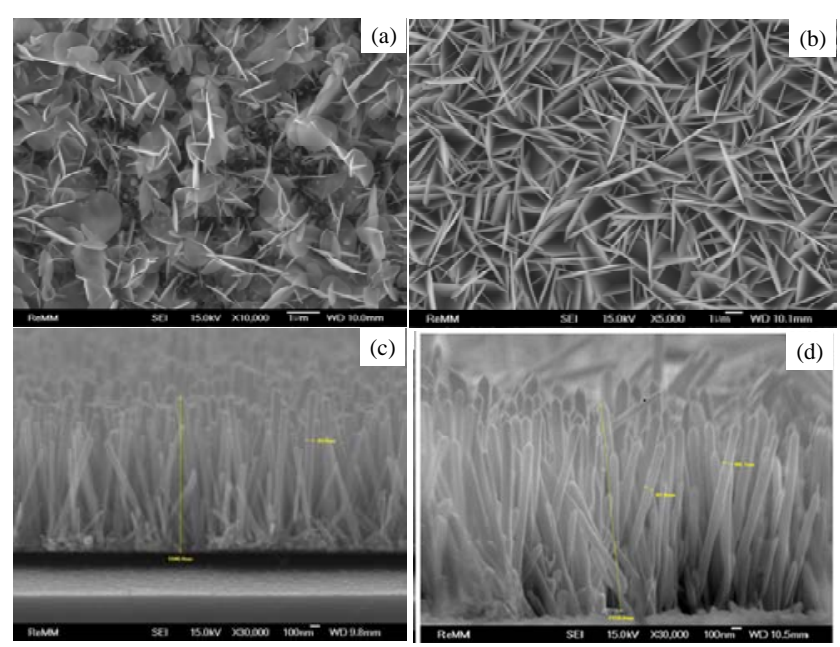

Fig. 2. FE-SEM images of GZO nanodisk (a, b) and GZO nanorods (c, d).

produces fine nanodisk morphology (Fig. 2(a) and (b)) with good film thickness and optimal crystallite size following annealing. The random/hybrid growth of nanorods/nanodisks is observed at low GZO concentration ( $0.2 \mathrm{~mol} / \mathrm{L})$, as shown in Fig. 2(c) and (d). Therefore, in the present study, a GZO concentration of $0.5 \mathrm{~mol} / \mathrm{L}$ was used for seed layer formation to obtain pure nanodisk or nanoflower morphology. Nanodisk formation depends on the interaction between surface seed layers of GZO particles and polymer molecules during hydrothermal treatment. We studied the effects of hydrothermal conditions and polymer concentration on the morphology of $(1 \%, 2 \%$, and $3 \%)$ GZO with a concentration of $0.5 \mathrm{~mol} / \mathrm{L}$ on AlN/Si substrates. The amount of gallium doped in GZO and the effect of polymer concentration are observed in FE-SEM images of the samples (Fig. 3). The optimal polymer concentration (4\%) in the hydrothermal process results in thinner nanodisks compared with those of samples prepared using different polymer concentrations. Fig. 3 shows that addition of $4 \%$ PEI produces nanodisks with thicknesses of around 96 and $102 \mathrm{~nm}$, respectively. The optimized polymer concentration gave thinner nanodisks than ones above $5 \%$, which resulted in the formation of aggregated thick nanodisks (Fig. 5). Therefore, we

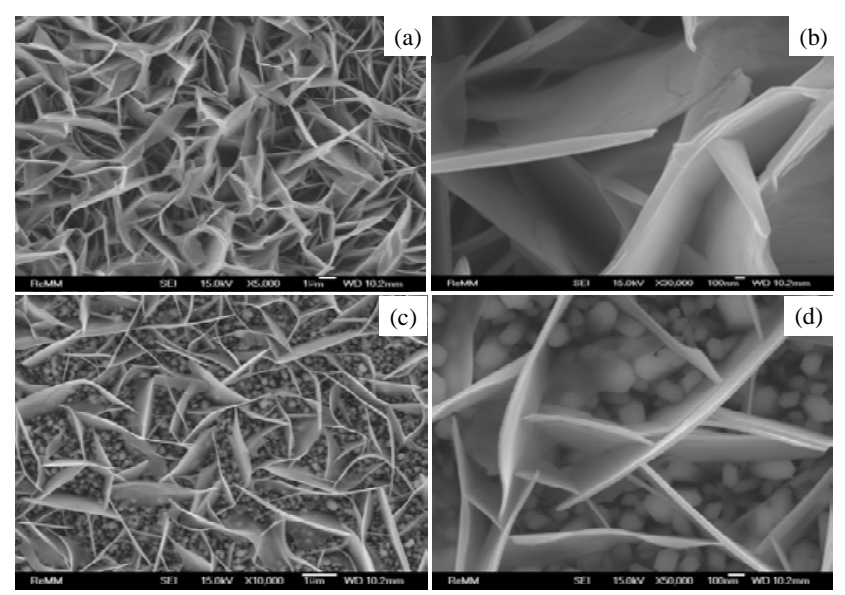

Fig. 3. FE-SEM images of $4 \%$ polymer assisted GZO nanodisk (a, b) and GZO nanodisk prepared using $0.2 \mathrm{~mol} / \mathrm{L} \mathrm{sol} \mathrm{solution} \mathrm{(c,} \mathrm{d).}$ 
used an optimized concentration of $3 \%$ or $4 \%$ polymer solution to produce GZO nanodisk and nanoflower structures by hydrothermal treatment.

Fig. 2 and Fig. 3 depict FE-SEM images of GZO nanodisks and nanorods prepared using optimized conditions such as 1\% GZO nanodisks formed using a polymer concentration of 3\% in the hydrothermal process. GZO nanorods were obtained when polymer was not included in the hydrothermal process under basic conditions. The present study aims to develop a polymer-assisted hydrothermal method for the formation of different nanostructures of GZO on piezoelectric substrates. Different tactics are used to form nanodisk and nanoflower morphologies. Nanodisk morphology was obtained using GZO solution $(0.5 \mathrm{~mol} / \mathrm{L})$ in the spin-coating process to make the GZO seed layer on the substrate, but not using a low concentration ( $0.2 \mathrm{~mol} / \mathrm{L})$ GZO solution. Fig. 3 shows the GZO sample prepared using $3 \%$ polymer in the hydrothermal process, which consists of uniform nanodisks. When the concentration of the polymer solution was increased to $4 \%$, thicker disks formed (Fig. 3(a) and (b)). Fig. 3(c) and (d) illustrate the results obtained using a low concentration of GZO $(0.2 \mathrm{~mol} / \mathrm{L})$ in the hydrothermal process. Both nanodisks and nanorods formed on the surface of the GZO seed layer on the substrate. We previously reported a detailed X-ray photoelectron spectroscopy (XPS) study about the role of (1\%, 2\% and 3\%) GZO in nanodisk formation [19]. Fig. 3 clearly indicates the nanorods form initially and are then transformed into the nanodisk structure. In the hydrothermal crystallization process, nanorods grow faster with the assistance of polymer molecules and aluminum species on the substrate, which are main driving forces for the morphology transformation from rods to disks.

Fig. 4 presents the GZO nanoflower morphology obtained using a vertically mounted substrate in the Teflon-lined autoclave during the hydrothermal process. The individual nanorods combine together to form the flower-like arrangement Higher-magnification SEM images (at the 1- $\mu \mathrm{m}$ scale) reveal that the flower-type GZO structures consist of hexagonal arrangements of smaller nanorods. Therefore, nanorod formation occurs first, and then the nanorods transform into other nanostructures depending on the reaction conditions. Fig. 5 compares FE-SEM images of GZO nanodisks on AlN/Si substrates formed using different polymer concentrations in the hydrothermal process. Fig. 5(a) and (b) show the GZO nanodisks prepared at the optimized concentration of 1\% GZO and polymer concentration of 3\%. Fig. 5(c) and (d) illustrate the

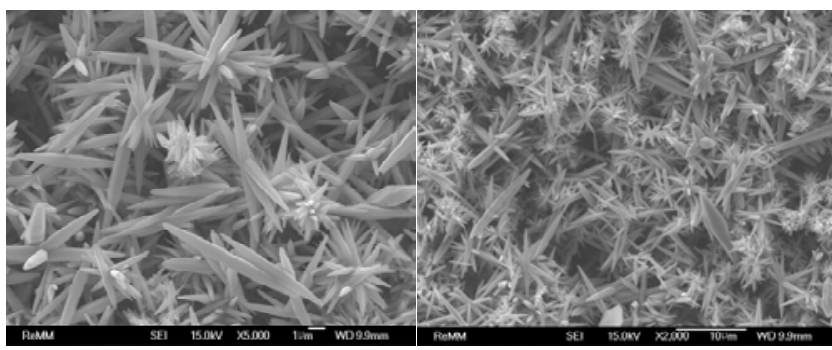

Fig. 4. FE-SEM images of GZO with nanoflower morphology
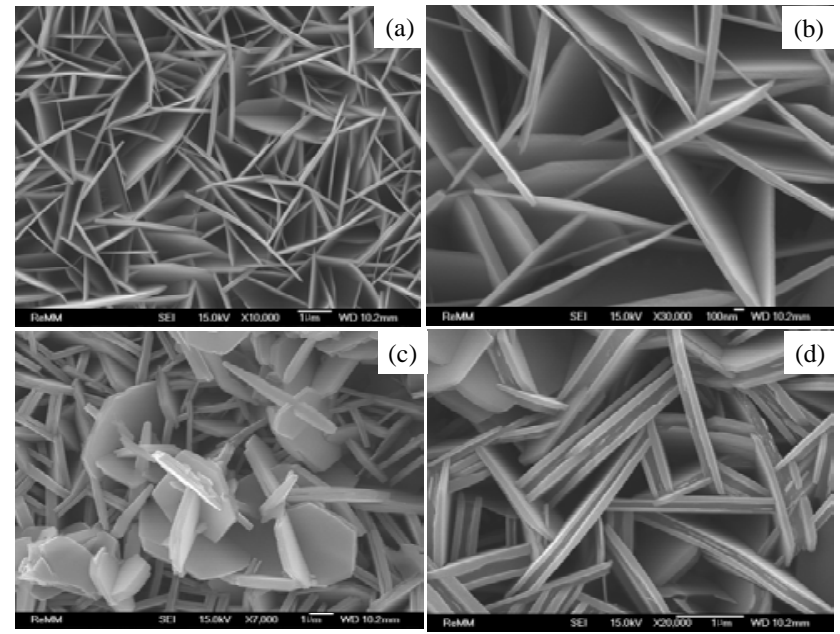

Fig. 5. FE-SEM images of GZO morphology. (a, b) GZO thin nanodisk; (c, d) Thick nanodisk.

result obtained using a polymer concentration of 5\%. Thicker nanodisks are formed at higher polymer concentration because the disks bind to each other when the polymer concentration is too high.

AFM was used to study the surface structure and roughness of the as-synthesized GZO nanostructured samples. The AFM images reveal clear differences in the surface structures of the samples. Figs. 6-8 present AFM images of the nanostructured GZO thin film. Fig. 6 shows GZO with nanorod morphology; these images were recorded at different magnification (ranges of 2.15 and $5.98 \mu \mathrm{m}$, respectively) with a surface height of 200 $\mathrm{nm}$. The nanorods adopted a cone-like array, which is clearly visible in Fig. 6. AFM images of GZO with nanodisk morphology are provided in Fig. 7. The nanodisks have a slightly different appearance to the nanorods. Each GZO nanodisk is a larger cone shape with different individual size to that of the nanodisks. Fig. 8 shows AFM images of GZO nanoflowers recorded at different magnification (ranges of 1.17 and $2.25 \mu \mathrm{m}$, respectively) with a surface height of $200 \mathrm{~nm}$.

Samples with rough or unique surface topography are promising for different applications like gas or liquid sensors and dye-sensitized solar cells [2,19]. ZnO has also been used as an ethanol sensor material. The micropores in $\mathrm{ZnO}$ surfaces are probably formed by the coalescence of small voids created by vaporization of residual organic species during crystallization in the hydrothermal process.

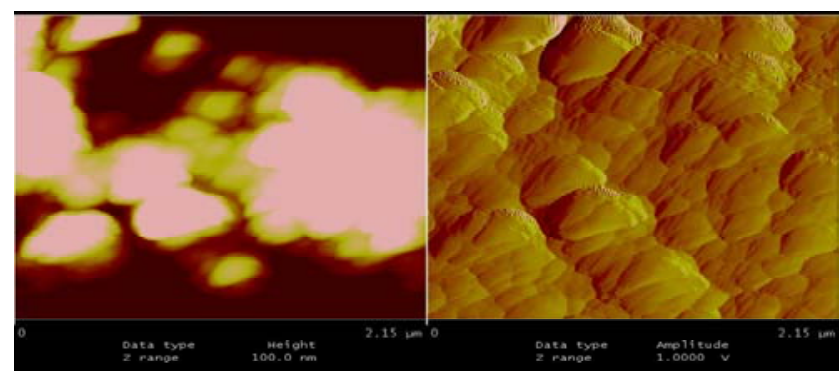

Fig. 6. AFM images of GZO nanorods. 


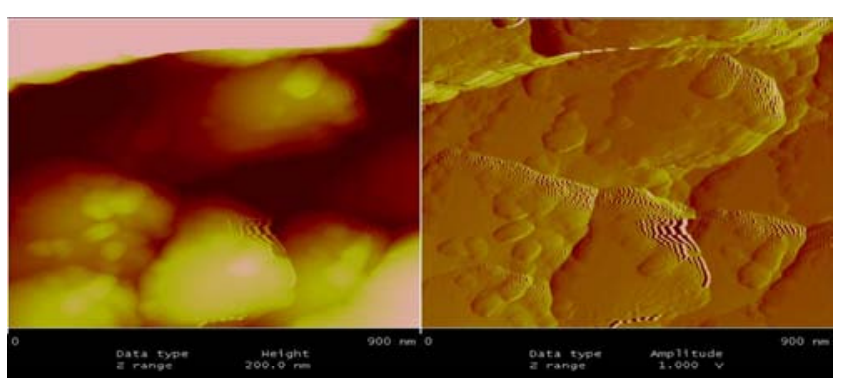

Fig. 7. AFM images of GZO nanodisks.

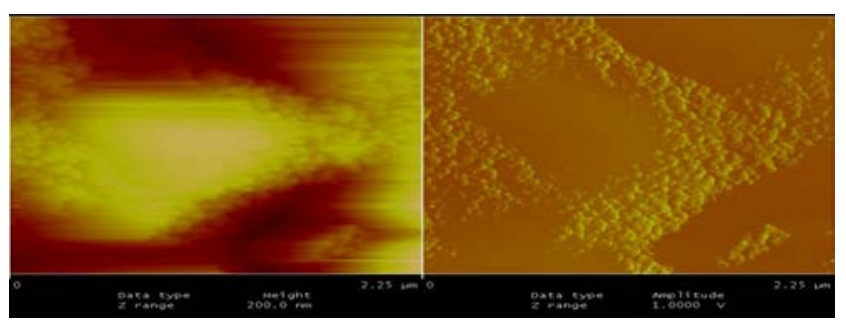

Fig. 8. AFM images of GZO nanoflowers.

Fig. 9 shows Raman spectra of GZO nanodisk and nanoflower structures together those of reference samples, including a conventional GZO thin film and ZnO powder. A sharp intense peak at $519 \mathrm{~cm}^{-1}$ is indexed as the transverse optical phonon mode of the silicon substrate [23]. Other than the major peak at $519 \mathrm{~cm}^{-1}$, the [E2 (high)-E2 (low)] mode of ZnO appeared at $330 \mathrm{~cm}^{-1}$ and the E2 high signal appeared at $437 \mathrm{~cm}^{-1}$ [24-26]. Fig. 9 illustrates the combined Raman spectra of GZO nanodisk and nanoflower samples with a pristine GZO peak at $577 \mathrm{~cm}^{-1}$ originating from the $\mathrm{A} 1$ longitudinal optical mode, but the peak at $577 \mathrm{~cm}^{-1}$ is not observed for the reference GZO and $\mathrm{ZnO}$ samples. Pure ZnO without any dopant synthesized by a conventional method displays the Raman shift values different from those of GZO. The Raman spectral data clearly distinguish the different surface properties of pristine and nanostructured GZO samples.

Fig. 10 shows interference contrast photographs and fluorescent images of GZO with different nanostructure morphologies. Fig. 10(a) and (b) reveal the florescence activity of GZO nanodisk samples under dark and fluorescence irradiation,

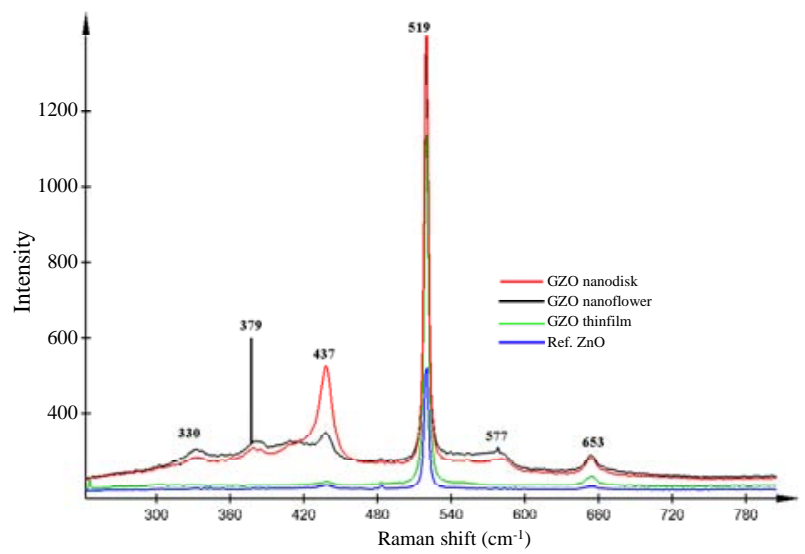

Fig. 9. Raman spectrum of GZO nanodisk, GZO nanoflowers, GZO thin film, and $\mathrm{ZnO}$.
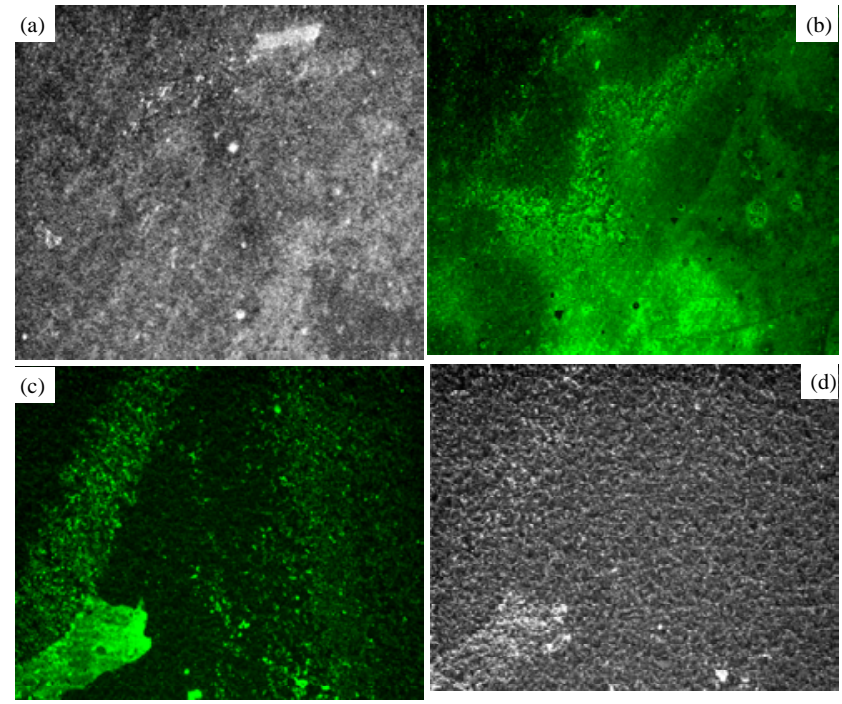

Fig. 10. Fluorescence images of GZO nanodisk $(a, b)$ and nanoflowers (c, d) with contrast photographs.

respectively. The fluorescence-active GFP site on the surface of $\mathrm{ZnO}$ emits green light under dark conditions. This clearly indicates the sensing activity of GFP immobilized on the GZO nanostructures under UV light irradiation. Similar fluorescence activity results are observed for the GZO nanoflowers, as illustrated in Fig. 10 (c) and (d).

The green emission from the protein-immobilized GZO nanostructure was clearly observed under a fluorescent microscope, indicating that the protein molecules are strongly attached to the GZO nanostructures. The interaction between nanostructures and biomolecules (e.g., proteins) results in the appearance of a biological corona on the nanosurface [27]. The biological corona can lower the photoluminescence of particles because of the conformation change and steric hindrance induced by the nanostructured surface.

The morphology of the products strongly depended on the concentrations of zinc precursor solution and polymer. GZO nanostructures are generally formed as layers by the step-by-step growth mechanism. Each ZnO nucleus individually grows along the $c$-axis into a rod-like shape initially and then evolves into different structures such as nanodisks or nanoflowers depending on the arrangement of individual rods of $\mathrm{ZnO}$ nuclei, which is influenced by reaction and substrate conditions. The $\mathrm{ZnO}$ surface has both positive and negative charges and the surface attracts ions of opposite charges from the solution and polymer, resulting in the formation of nanostructure morphology. The presence of AlN on the Si substrate causes the rods to transform into nanodisk morphology along the $c$-axis perpendicular to the surface. Aluminum particles act as a catalyst for nanostructure morphology formation by altering the rod shape of $\mathrm{ZnO}$ nuclei, and the polymer molecules further assist the shape formation during the hydrothermal process.

Finally, the sensing activity of the as-prepared GZO nanostructured materials was characterized by UV light sensing test reactions. The sensing response of GFP-immobilized 


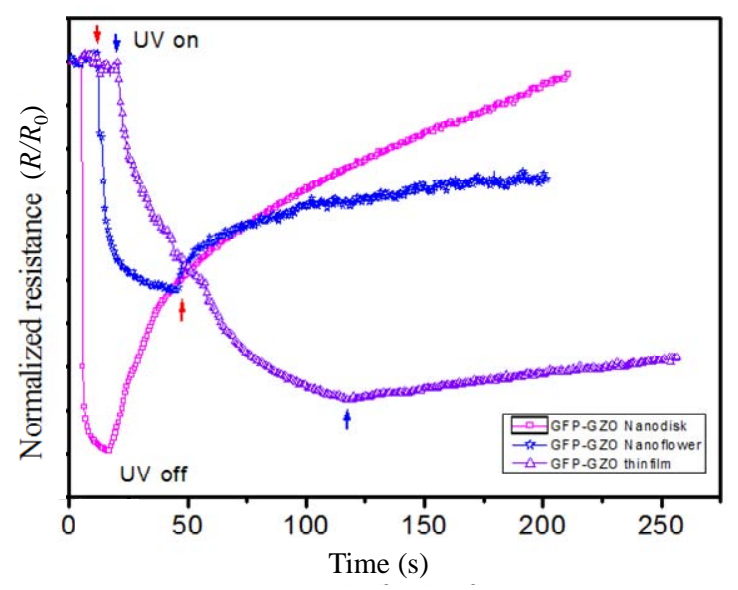

Fig. 11. UV-light sensor analysis of pristine GZO, GZO nanodisk and GZO nanoflower.

GZO nanodisk and nanoflower samples was analyzed in the dark ( $30 \mathrm{~s}$ each cycle) and in the presence of UV irradiation (10 s each cycle). Fig. 11 shows the UV light sensing activity of GZO nanodisks, which displayed a good response and reproducible recovery time. The GZO nanoflower structure exhibited lower activity than the GZO nanodisks when the same amount of GFP was immobilized on the surface of each GZO sample. Meanwhile, GFP-immobilized undoped GZO (formed without polymer addition during hydrothermal treatment) showed a poor response to UV light compared with that of the GZO samples with nanostructured morphology.

\section{Conclusions}

GZO samples with different morphologies such as nanodisk and nanoflower structures were prepared on piezoelectric substrate using an optimized hydrothermal process involving polymer assistance. Commercially available GFP was immobilized on the GZO nanostructures to produce sensors for bioimaging applications. XRD patterns confirmed the formation of wurtzite-phase GZO nanodisk and nanoflower structures. The optimal concentration of GZO (0.5 mol/L) solute gave nanodisk and nanoflower morphologies under suitable reaction conditions. The effects of Ga doping concentration and polymer concentration on GZO nanostructure morphology was studied by FE-SEM. A high polymer concentration in the hydrothermal process resulted in aggregated nanodisk morphology. AFM images revealed the surface structures of various GZO nanostructures. Fluorescence images and contrast photographs confirmed that GFP bound to the GZO nanostructures. The active sites of the GFP-immobilized GZO nanostructures emitted green light upon irradiation of the samples with UV light. The UV light sensing activity of the GFP-immobilized GZO nanodisk structure was higher than that of GZO nanoflower and undoped GZO samples. Our results reveal that GZO with specific morphologies can display specific activity for UV light-assisted sensor fabrication.

\section{Acknowledgments}

This work was supported by King Saud University, Vice Deanship of Research Chairs.

\section{References}

[1] N. G. Nguyen, V. T. Thanh Ho, L. S. Hong, Appl. Phys. Lett., 2013, 102, 181912/1-181912/4.

[2] M. Gabas, P. Díaz-Carrasco, F. Agullo-Rueda, P. Herrero, A. R. Landa-Canovas , J. R. Ramos-Barrado, Solar Energy Mater. Solar Cells, 2011, 95, 2327-2334.

[3] X. H. Gao, J. L. Zhang, N. Chen, Q. X. Ma, S. B. Fan, T. S. Zhao, N. Tsubaki, Chin. J. Catal., 2016, 37, 510-516.

[4] T. J. Hsueh, C. L. Hsu, S. J. Chang, I. C. Chen, Sensors Actuat. B, 2007, 126, 473-477.

[5] M. Ahmad, E. Ahmed, W. Ahmed, A. Elhissi, Z. L. Hong, N. R. Khalid, Ceram. Int. A, 2014, 40, 10085-10097.

[6] J. X. Wang, X. W. Sun, A. Wei, Y. Lei, X. P. Cai, C. M. Li, Z. L. Dong, Appl. Phys. Lett., 2006, 88, 233106/1-233106/3.

[7] A. Wei, X. W. Sun, J. X. Wang, Y. Lei, X. P. Cai, C. M. Li, Z. L. Dong, W. Huang, Appl. Phys. Lett., 2006, 89, 123902/1-123902/3.

[8] L. Y. Chen, B. X. Gu, G. P. Zhu, Y. F. Wu, S. Q. Liu, C. X. Xu, Nano: Brief

\section{Graphical Abstract}

Chin. J. Catal., 2016, 37: 1235-1241 doi: 10.1016/S1872-2067(16)62464-6

\begin{abstract}
Preparation and surface characterization of nanodisk/nanoflower-structured gallium-doped zinc oxide as a catalyst for sensor applications

R. Jothi Ramalingam*, T. Radika, Hamad A. Al-Lohedan King Saud University, Saudi Arabia; Centre for Materials Electronic Technology, India; University of Ulsan, Korea
\end{abstract}

Gallium doped $\mathrm{ZnO}$ in the form thin films with different morphologies has been prepared by adopting suitable hydrothermal reaction condition. In the second stage, green fluorescence protein immobilized on as prepared thin film.

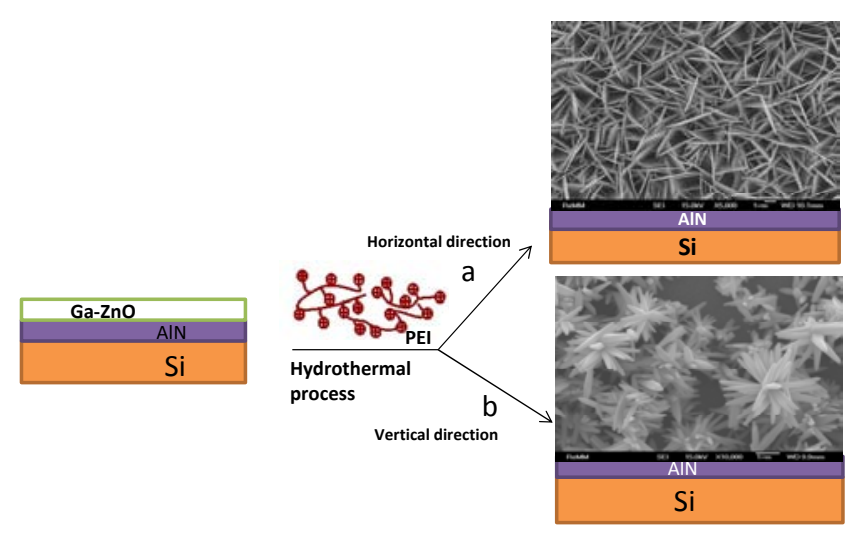


Rep. Rev., 2007, 2, 281-284.

[9] Y. L. Wu, C. S. Lim, S. Fu, A. I. Y. Tok, H. M. Lau, F. Y. C. Boey, X. T. Zeng, Nanotechnology, 2007, 18, 215604/1-215604/9.

[10] Y. L. Wu, S. Fu, A. I. Y. Tok, X. T. Zeng, C. S. Lim, L. C. Kwek, F. C. Y. Boey, Nanotechnology, 2008, 19, 345605/1-345605/9.

[11] X. S. Tang, E. S. G. Choo, L. Li, J. Ding, J. M. Xue, Chem. Mater., 2010, 22, 3383-88.

[12] X. S. Tang, E.S. G. Choo, L. Li, J. Ding, J. M. Xue, Langmuir, 2009, 25, 5271-5275.

[13] R. O. Moussodia, L. Balan, C. Merlin, C. Mustin, R. Schneider, J. Mater. Chem., 2010, 20, 1147-1155.

[14] H. M. Xiong, Y. Xu, Q. G. Ren, Y. Y. Xia, J. Am. Chem. Soc., 2008, 130, 7522-7523.

[15] K. Senthilkumar, O. Senthilkumar, K. Yamauchi, M. Sato, S. Morito, T. Ohba, M. Nakamura, Y. Fujita, Phys. Status Solidi (B), 2009, 246, 885-888.

[16] M. Sato, H. Harada, S. Morito, Y. Fujita, S. Shimosaki, T. Urano, M. Nakamura, Appl. Surf. Sci., 2010, 256, 4497-4501.

[17] S. Saliba, C. V. Serrano, J. Keilitz, M. L. Kahn, C. Mingotaud, R. Haag, J. D. Marty, Chem. Mater., 2010, 22, 6301-6309.
[18] H. M. Xiong, D. P. Xie, X. Y. Guan, Y. J. Tan, Y. Y. Xia, J. Mater. Chem., 2007, 17, 2490-2496.

[19] P. Thirunavukarasu, R. Jothi Ramalingam, Powder Technol., 2013, 239, 308-313.

[20] N. Barie, M. Rapp, Biosens. Bioelectron., 2001, 16, 979-981.

[21] R. Xu, Y. D. Jiang, L. Xia, T. X. Zhang, L. Xu, S. Zhang, D. L. Liu, H. W. Song, Biosens. Bioelectron., 2015, 74, 411-417.

[22] K. Brince Paul, S. Kumar, S. Tripathy, S. R. K. Vanjari, V. Singh, S. G. Singh, Biosens. Bioelectron., 2016, 80, 39-46.

[23] M. Hirasawa, T. Orii, T. Seto, Appl. Phys. Lett., 2006, 88, 093119/1-093119/3.

[24] A. El Manouni, F. J. Manjon, M. Mollar, B. Marí, R. Gomez, M. C. Lopez, J. R. Ramos-Barrado, Superlatice. Microst., 2006, 39, 185-192.

[25] S. L. Mensah, V. K. Kayastha, I. N. Ivanov, D. B. Geohegan, Y. K. Yap, Appl. Phys. Lett., 2007, 90, 113108/1-113108/3.

[26] A. Umar, B. Karunagaran, E. K. Suh, Y. B. Hahn, Nanotechnology, 2006, 17, 4072-4077.

[27] M. Mahmoudi, I. Lynch, M. R. Ejtehadi, M. P. Monopoli, F. B. Bombelli, S. Laurent, Chem. Rev., 2011, 111, 5610-5637.

\title{
传感器用镓掺杂氧化锌纳米盘/纳米花状结构催化剂的制备及表面表征
}

\author{
R. Jothi Ramalingam ${ }^{\text {a,c, }}$, T. Radika b, Hamad A. Al-Lohedan ${ }^{\text {a }}$ \\ a 沙特阿拉伯国王大学理学院化学系, 表面活性剂研究中心, 利雅得11451, 沙特阿拉伯 \\ ${ }^{\mathrm{b}} \mathrm{卩}$ 度电子技术材料研究中心, 特里苏尔, 喀拉拉邦, 印度 \\ ‘蔚山大学工程学院, 韩国
}

摘要: 采用简便的旋涂过程和一步水热法在压电基片上制备了 Ga掺杂的ZnO纳米薄膜(GZO). 在水热处理过程中, 通过添 加不同的聚合物可形成纳米盘和纳米花状形貌的薄膜. 采用场发射扫描电镜(Fe-SEM)、X射线衍射(XRD)和Raman光谱表 征了样品的形貌、微结构和组成. XRD和FE-SEM结果证明, 在AIN/Si压电基片上形成的纳米盘、纳米棒和纳米花状GZO 均为纤维锌矿相. 采用浸渍法进一步在所制GZO样品上固定了绿色的荧光蛋白质(GFP). 运用原子力显微镜和苂光光谱分 析了GFP与GZO表面结合的性质, 考察了其用于传感器和生物成像技术的可行性. 痕量GFP的固定使该材料产生荧光响应, 表明其用于紫外光传感器时具有较好活性.

关键词: 半导体; 薄膜; 溶胶凝胶生长; 原子力显微镜; 导电性; 表面性质

收稿日期: 2016-01-24. 接受日期: 2016-05-09. 出版日期: 2016-08-05.

*通讯联系人. 电话: +966-553510565; 传真: +966-1-4679972; 电子信箱: jrajabathar@ksu.edu.sa

本文的英文电子版由Elsevier出版社在ScienceDirect上出版(http://www.sciencedirect.com/science/journal/18722067). 